\title{
Automation of Finite Element Aided Design of Induction Motors Using Multi-Slice 2D Models
}

\author{
D R Griffiths ${ }^{1}$ and J K Sykulski ${ }^{2}$ \\ ${ }^{1}$ FR-HiTEMP Limited,Titchfield, Fareham, PO14 4QA, UK, E-mail: David.Griffiths@fr-hitemp.co.uk \\ ${ }^{2}$ Electrical Power Engineering, School of ECS, \\ University of Southampton, Southampton SO17 1BJ, UK, E-mail: jks@soton.ac.uk
}

\section{GENERAL}

This paper describes the way in which a generalpurpose 2D electromagnetic finite element analysis software package OPERA [1] has been used - in conjunction with Matlab programming language and Microsoft Excel - to perform automated simulation and performance analysis of induction motors as part of the design and optimisation process. The objective of this work is to develop a practical tool for design purposes where full 3D transient solutions are not acceptable due to excessive computing times. It is also the requirement that a commercial FE package has to be used and some 3D effects, in particular those due to rotor bar skew, are taken into account. Finally, a steady-state (single harmonic) FE solution is utilised for performance prediction

\section{INTRODUCTION}

Basic design data in parametric form is processed within the main controlling program in an automated fashion to generate data suitable for use in the selected FE software (which is invoked as a subroutine from the main program). There appears to be little information available in literature on development of practical systems utilising commercially available software where 3D effects are incorporated into 2D analysis. Thus the discussion of practical aspects of 2D FE modelling will be further developed to include the impact of 3D effects. In particular the effects of the rotor bar skew are considered and the possibility of their inclusion within the 2D environment. The approach employed is set against the background of other similar techniques reported in literature since the early 1990's [2]-[3].

\section{DESCRIPTION}

The overall concept and basic structure of the software is shown in Fig 1. A user friendly GUI is currently under development to permit easier management of data input and transfer. The automated program controlling the preand post-processing of the FE models and calculating the performance characteristics of the machine is completed. The main features of the system are a central controlling routine that initiates paths, performs data manipulation, creates buffer files to interface between the commercial software packages and launches a solver. The system described forms a core of a design/optimisation platform to be used in design environment.
Parametric data characterising the machine to be analysed is entered and includes electrical, geometric and materials data. This is automatically processed and checked via Matlab routines and subsequently output into several data files for access by the FE software. For performance prediction the software's main Matlab solver routine is called. The routine completes the pre-processing commensurate with the slot design and number of slices and launches the FE software to create a model ready for analysis. With the model generated and saved, a second call to the FE software initiates a time stepping analysis through one slot pitch. Results from the analysis are processed taking account of the number of slices and time steps used; the final compound results are transferred to Excel. The numeric output provides details of typical performance parameters, such as currents, torque, power factor, etc, for each point at given speed.

\section{RESULTS}

The torque/speed characteristic and other output parameters obtained using different number of slices (from 1 to 5) from the 2D model have been compared against similar curves obtained from a 3D model and those established experimentally. The comparison will be discussed in some detail in the full version of the paper.

\section{CONCLUSIONS}

The software developed through the use of three commercial packages to form a single practical design tool, and thus providing designers with easier access to $\mathrm{FE}$ analysis, has been introduced in this paper. Main conclusions will consider the applicability and accuracy of the proposed approach.

\section{REFERENCES}

[1] PC-OPERA, "Reference Manual", Vector Fields Ltd., Oxford, UK.

[2] Tenhunen A., Arkkio A., "Modelling of Induction Machines With Skewed Rotor Slots", IEE Procedures, vol. 148. No 1, January 2001.

[3] Ho S.L., Fu W.N., "A Comprehensive Approach to the Solution of Direct -Coupled Multislice Model of Skewed Rotor Induction Motors Using Time-Stepping EddyCurrent Finite Element Method", IEEE Transactions on Magnetics Vol. 33, No 3 May 1997, pp 2265 - 2273.

[4] Al-Khoury A.H., Sykulski J.K., "Automation of Finite Element Aided Design of Brushless PM Motors", International Conference Electrical Machines (ICEM 98), vol. 1, pp. 614-618, September 1998, Istanbul, Turkey. 


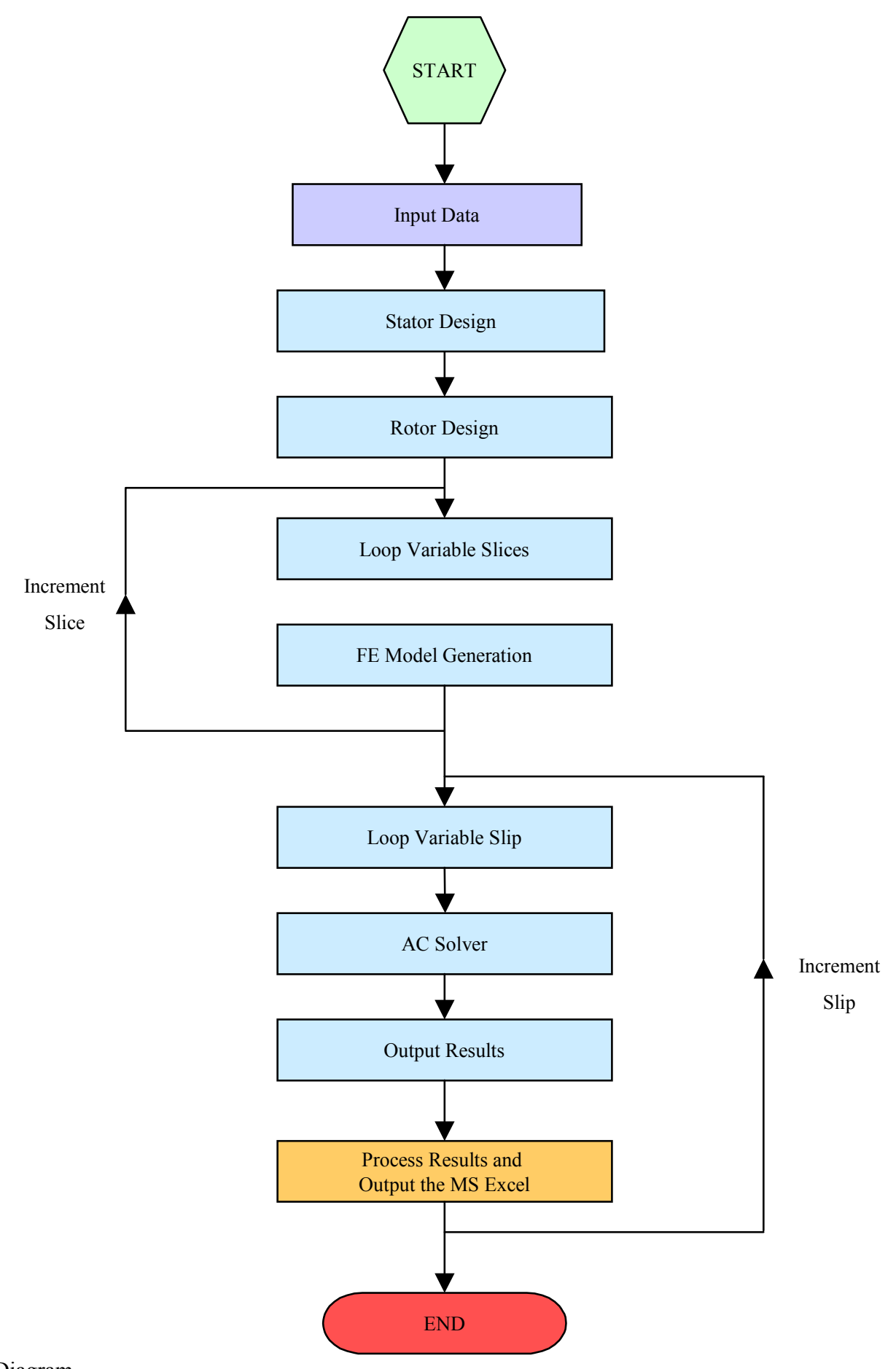

Figure 1. Block Diagram 